\title{
Editorial \\ The Global Strategy for the Management of Farm Animal Genetic Resources - an Update
}

The FAO programme entity Global Strategy for the Management of Farm Animal Genetic Resources is to help tap the significant potential of animal genetic resources to both food security and rural economic diversification and development. To realize this potential, the use and development of animal genetic resources must be effectively planned to achieve the desirable outcomes. Past attempts to increase agricultural production using genetic improvement of domestic animals have not always been well planned and undertaken with all of the important factors to be considered. Locally adapted breeds are often able to survive and produce valuable products in low input and variable environments. A strategy to develop them is likely to be more sustainable over the long-term than reliance external genetic resources.

Animal genetic diversity is rapidly eroding despite the strongly growing demand for livestock products. Conservation and development of animal genetic resources is essential to enable farmers to adapt to changing environmental conditions and consumer demands.

FAO, in using its comparative advantages, is leading efforts to sustainably use, develop, and conserve animal genetic resources, and since 1993 has been engaged in the preparation of the Global Strategy for the Management of Farm Animal Genetic Resources. The Global Strategy is intended to serve as a strategic framework to guide international efforts in the animal genetic resources sector. Preparation of the First Report on the State of the World's Animal Genetic Resources will be an essential element of the Global Strategy up to 2004-2005. There is a specific clear mandate from FAO member countries through the Commission on Genetic Resources for Food and Agriculture to develop the Global Strategy for the Management of Animal Genetic Resources along the lines followed by this entity. Further priority actions for the sustainable use of farm animal genetic diversity will be based on the First Report. The proposed Report is a country-driven process, endorsing FAO's guidelines for the preparation of country reports. Furthermore, the FAO Working Group on Animal Genetic Resources requested that countries, donors, stakeholders and FAO increase efforts to mobilize the resources necessary, including financial resources, in order to successfully undertake the preparation of the Report and implement priority follow-up action. FAO stands ready to assist its member nations in the preparation of this landmark document and in implementing country assistance for priority actions in conservation and management of farm animal genetic resources. The country driven State of the World's Animal Genetic Resources process has already been initiated with the FAO Director-General inviting countries to participate in this global effort. To date 103 countries have formally accepted. A set of training and reporting tools has been developed to assist countries in preparing their national reports. A global training effort has been implemented, reaching 123 countries and 224 professionals in 2001, and ending up in May 2002 by reaching 53 additional countries and 120 more professionals. This undertaken is expected to go on to the year 2005 until the technical and analytical work 
related to Report has been completed and will expectedly cost US\$ 3400000 of

extra-budgetary funds.

This activity will contribute to the integration of the management of animal genetic resources into other agricultural activities and will lead to the enhancement of capacities of communities to utilize local animal genetic resources for sustainable rural livelihoods, as it will address more equitable access to resources and helps develop international instruments to regulate global animal genetic resources. The activity also relates to other activities of concern to FAO like Biological Diversity, Biotechnology, Ethics and Sustainable Livelihoods.

The Editors 


\section{Editorial \\ Stratégie Mondiale pour la Gestion des Ressources Génétiques des Animaux Domestiques - Mise à Jour}

Le programme de la FAO pour la Stratégie mondiale pour la gestion des ressources génétiques des animaux domestiques vise à exploiter l'important potentiel de ressources génétiques animales tant pour atteindre la sécurité alimentaire que pour la diversification et développement de l'économie rurale. Pour atteindre ce but, l'utilisation et le développement des ressources génétiques animales doivent être planifiés de façon effective. Les actions entreprises par le passé pour augmenter la production agricole au moyen de l'amélioration génétique des animaux domestiques n'ont pas toujours été bien planifiées ni réalisées en tenant compte de tous les facteurs nécessaires. Les races locales adaptées sont souvent capables de survivre et de fournir des productions valables avec un minimum d'intrants et dans des conditions environnementales variables. Une stratégie orientée vers le développement de ces races serait plus durable à long terme plutôt que de recourir à des ressources génétiques externes.

La diversité génétique animale subit une érosion rapide malgré la forte demande croissante de produits animaux. La conservation et le développement des ressources génétiques animales sont essentiels pour permettre aux éleveurs de s'adapter aux changements des conditions de milieu et de la demande des consommateurs.

La FAO, grâce aux avantages dont elle dispose, dirige les efforts pour une utilisation, un développement et une conservation des ressources génétiques animales durables. Pour cela, depuis 1993, la FAO a entrepris la préparation de la Stratégie mondiale pour la gestion des ressources des animaux domestiques qui doit servir comme cadre stratégique d'orientation des efforts internationaux dans le secteur des ressources génétiques animales. La préparation du Premier rapport sur la Situation mondiale des ressources génétiques animale sera un des éléments essentiels de la Stratégie mondiale pour la période 2004-2005. Il existe un mandat spécifique des pays membres de la FAO à la Commission pour les ressources génétiques pour l'alimentation et l'agriculture afin de développer la Stratégie mondiale pour la gestion des ressources génétiques des animaux domestiques suivant les lignes préconisées par cette entité. Les actions ultérieures pour l'utilisation durable des la diversité génétique des animaux domestiques feront référence à ce Premier rapport. Ce Rapport doit être le résultat d'une procédure nationale de démarrage qui accepte les normes de la FAO pour la préparation du rapport national. En outre, le Groupe de travail de la FAO pour les Ressources génétiques animales a demandé aux pays, aux donateurs, aux parties prenantes et à la FAO d'augmenter leurs efforts pour mobiliser les ressources nécessaires, y compris des ressources financières, afin de pouvoir commencer avec succès la préparation du Rapport et mener à terme les actions de suivi prioritaires. La FAO est prête à donner son assistance aux pays membres pour la préparation de ce document général et pour la réalisation des actions prioritaires nécessaires à la conservation et la gestion des ressources génétiques des animaux domestiques. La procédure nationale sur la Situation mondiale des ressources génétiques animales a déjà été lancée avec l'invitation du Directeur général aux pays membres pour qu'ils prennent part 
à cet effort global. Jusqu'à présent 103 pays ont confirmé officiellement leur adhésion. Une série d'outils pour la formation et la préparation du rapport ont été développées pour aider les pays dans la préparation de leur rapport national. Un effort global pour la formation a été réalisé et a bénéficié à 123 pays et 224 professionnels en 2001, et 53 pays et 120 professionnels jusqu'en mai 2002. Cet effort devrait continuer jusqu'en 2005, jusqu'à la finalisation du travail technique et analytique du Rapport, et ceci avec un coût prévu de US\$ 3400000 en provenance des fonds extra budgétaires.

Cette activité contribuera à l'intégration de la gestion des ressources génétiques animales dans d'autres activités agricoles et renforcera les capacités des communautés rurales à utiliser les ressources génétiques animales locales pour subvenir durablement à leurs besoins, étant donné qu'elle permettra un accès plus équitable aux ressources et aidera le développement des moyens internationaux pour réglementer les ressources génétiques animales mondialement. Cette activité a aussi des liens avec d'autres activités qui concernent la FAO, telles que la diversité biologique, la biotechnologie, l'éthique et les moyens d'existence durables. 


\section{Editorial \\ Estrategia Mundial para la Gestión de los Recursos Zoogenéticos - Actualización}

El programa de la FAO para la Estrategia mundial para la gestión de los recursos zoogenéticos fomenta en un primer momento la importancia del potencial de los recursos zoogenéticos con el fin de alcanzar la seguridad alimentaria y mantener la diversificación y el desarrollo de la economía rural. Para alcanzar este objetivo, la utilización y el desarrollo de los recursos zoogenéticos deben ser planificados de forma efectiva. Las acciones llevadas a cabo en el pasado para aumentar la producción agrícola a través el uso de la mejora genética de los animales domésticos no siempre fueron planificadas correctamente ni llevadas a cabo teniendo en cuenta todos los factores implicados. Las razas locales adaptadas son a menudo capaces de sobrevivir y proporcionar productos válidos con un mínimo de aportes y en condiciones ambientales variables. Una estrategia orientada hacia el desarrollo de estas razas sería más sostenible a largo plazo en vez de cubrir simplemente las necesidades nacionales con recursos genéticos externos.

La diversidad genética animal presenta una erosión rápida a pesar de la fuerte demanda creciente de productos animales. La conservación y el desarrollo de los recursos genéticos animales son esenciales para permitir a los agricultores adaptarse a los cambios de condiciones del ambiente y de la demanda de los consumidores.

La FAO, gracias a las ventajas de que dispone, dirige sus esfuerzos hacia una utilización, un desarrollo y una conservación sostenible de los recursos zoogenéticos . Para ello, y desde 1993, la FAO ha dado inicio a la preparación de la Estrategia mundial para la gestión de los recursos zoogenéticos de los animales domésticos que deberá servir de marco estratégico de orientación a los esfuerzos internacionales dentro del sector de los recursos zoogenéticos . La preparación del Primer informe sobre la Situación mundial de los recursos genéticos animales será uno de los elementos esenciales de la Estrategia mundial para el período 2004-2005. Existe un mandato específico de los países miembros de la FAO a la Comisión para los recursos genéticos para la alimentación y la agricultura, para que desarrolle la Estrategia mundial para la gestión de los recursos genéticos de los animales domésticos en base a las normas establecidas por esta entidad. Las acciones posteriores para el uso sostenible de la diversidad genética de los animales domésticos harán referencia a este Primer informe. Este Informe debe ser el resultado de un acuerdo nacional inicial que acepte las normas de la FAO para la preparación del informe nacional. En un segundo momento, el Grupo de trabajo de la FAO para los Recursos zoogenéticos solicitará a estos países, a los donantes, a los agricultores, a los ganaderos y a la FAO, que aumenten sus esfuerzos para movilizar los recursos necesarios, incluidos los financieros, para poder iniciar con éxito la preparación del Informe y llevar a cabo las actividades prioritarias de seguimiento. La FAO está dispuesta a aportar su asistencia a los países miembros en la preparación de este documento general y en la realización de las actividades prioritarias necesarias para la conservación y la gestión de los recursos zoogenéticos. El informe nacional sobre la Situación mundial de los recursos genéticos animales ha iniciado ya con la invitación del Director general a los países miembros para que tomen parte en este esfuerzo global. 
Hasta hoy, 103 países han confirmado oficialmente su adhesión. Se ha puesto a punto una metodología para la formación y la preparación del informe, y ayudar a los países en la preparación del informe nacional. También se ha realizado un esfuerzo global de formación que ha beneficiado en 2001 a 123 países y 224 profesionales, y 53 países y 120 profesionales hasta mayo 2002. Esta situación debería seguir hasta el 2005, es decir, hasta la finalización del trabajo técnico y analítico del Informe, y esto con un costo previsto de \$EE.UU. 3400000 con cargo a los fondos provenientes de los presupuestos extraordinarios.

Esta actividad contribuirá en la integración de la gestión de los recursos genéticos animales con otras actividades agrícolas y llevará a fomentar las capacidades de las comunidades en la utilización de los recursos genéticos de animales locales a través de medios de existencia rurales sostenibles, dado que permitirá un acceso más equitativo a los recursos y ayudará al desarrollo de los medios internacionales para normalizar los recursos zoogenéticos globalmente. El informe se refiere también a otros actividades que conciernen a la FAO, tales como la biodiversidad biológica, la biotecnología, la ética y los medios de subsistencia sostenibles. 